\title{
A Scalable and Accurate Feature Representation Method for Identifying Malicious Mobile Applications
}

\author{
Bo Sun \\ National Institute of Information and \\ Communications Technology \\ Tokyo, Japan \\ bo_sun@nict.go.jp \\ Yeali S.Sun \\ Department of Information \\ Management, National Taiwan \\ University \\ Taipei, Taiwan \\ sunny@ntu.edu.tw
}

\author{
Tao Ban \\ National Institute of Information and \\ Communications Technology \\ Tokyo, Japan \\ bantao@nict.go.jp \\ Takeshi Takahashi \\ National Institute of Information and \\ Communications Technology \\ Tokyo, Japan \\ takeshi_takahashi@nict.go.jp
}

\author{
Shun-Chieh Chang \\ Cybersecurity Technology Institute, \\ Institute for Information Industry \\ Taipei, Taiwan \\ scchang@iii.org.tw \\ Daisuke Inoue \\ National Institute of Information and \\ Communications Technology \\ Tokyo, Japan \\ dai@nict.go.jp
}

\begin{abstract}
With the dramatic growth in smartphone usage, the number of new malicious mobile applications has increased rapidly. Identifying malicious applications in large-scale datasets is intensive and time consuming. Multiple previous studies have focused on automating the process of malicious application detection using machine (or deep) learning technology. However, a scalable and accurate solution is still lacking for large-scale applications. Therefore, in this study, we propose a novel approach to improve the accuracy of discovering malicious applications and decrease the computation time for processing the analysis. We implemented our proposed approach combining data collection, static feature extraction, and machine learning algorithms. Using a large dataset collected from a mobile application store that included 49,045 benign samples and 12,685 malicious samples, we demonstrate that the F-measure of the malicious application detection of our approach ranges from 0.968 to 0.995 , with a false positive rate of $0.48 \% \sim 3.3 \%$. We find that a multi-layer perceptron classifier performs best in these algorithms. Moreover, the analysis processing running time can be compressed to less than $18 \mathrm{~min}$. Finally, we compare our method to those of two types of previous studies and report a better performance in terms of scalability and accuracy.
\end{abstract}

\section{CCS CONCEPTS}

- Information systems $\rightarrow$ Document representation; • Security and privacy $\rightarrow$ Malware and its mitigation; Mobile platform security; • Computing methodologies $\rightarrow$ Neural networks; Supervised learning;

Permission to make digital or hard copies of all or part of this work for personal or classroom use is granted without fee provided that copies are not made or distributed for profit or commercial advantage and that copies bear this notice and the full citation on the first page. Copyrights for components of this work owned by others than ACM must be honored. Abstracting with credit is permitted. To copy otherwise, or republish, to post on servers or to redistribute to lists, requires prior specific permission and/or a fee. Request permissions from permissions@acm.org.

SAC'19, Limassol, Cyprus

(C) 2019 Association for Computing Machinery.

ACM ISBN 978-1-4503-5933-7/19/04 . \$ \$15.00

https://doi.org/10.1145/3297280.3297396

\section{KEYWORDS}

Malicious mobile application, Machine learning, Deep learning, Natural language processing, Semantic-aware

ACM Reference Format:

Bo Sun, Tao Ban, Shun-Chieh Chang, Yeali S.Sun, Takeshi Takahashi, and Daisuke Inoue. 2019. A Scalable and Accurate Feature Representation Method for Identifying Malicious Mobile Applications. In The 34th ACM/SIGAPP Symposium on Applied Computing (SAC '19), April 8-12, 2019, Limassol, Cyprus. ACM, New York, NY, USA, Article 4, 8 pages. https://doi.org/10.1145/3297280. 3297396

\section{INTRODUCTION}

In recent years, smartphones have completely infiltrated our daily lives; accordingly, smartphones have become one of the main targets for attackers. McAfee [3] reported that approximately 2 million new malicious mobile applications were discovered in only the first quarter of 2018. Such dramatically increasing numbers of new malicious mobile applications have led to a heavy burden for security specialists in terms of manual analyses. Automating the analysis process for malicious mobile applications, therefore, has triggered a great deal of interest in the security research community.

To implement analysis automation, machine and deep learning ${ }^{1}$ are suitable technologies that have been applied in multiple previous studies. Traditional machine learning, such as support vector machine (SVM) and $\mathrm{K}$ nearest neighbor (KNN), are also mainstream approaches. Traditional machine learning-based approaches $[9,10,14,21,23,24,26]$ extract features from mobile application packages, such as application programming interface (API) calls and permission requests, or metadata in app stores, such as categories and descriptions, and then apply feature selection technology to determine the effective features and decrease the size of the feature vectors. Based on these selected features, traditional machine learning algorithms are used to conduct binary detections for mobile applications. With the rapid development and sophistication of deep learning, security researchers have started employing

\footnotetext{
${ }^{1}$ Deep learning is a border family of machine learning methods, In this paper, we distinguish deep learning from machine learning for the better understanding. The machine learning in this paper specifies the traditional algorithm such as SVM and KNN
} 
this new technology. Deep learning-based approaches [13, 19, 27] also need to extract features from mobile application packages or metadata; however, unlike traditional machine learning, deep learning can represent features automatically in an informative manner, instead of via feature selection.

The number of mobile applications has continued to increase dramatically over time. Detecting malicious mobile applications in large-scale data, therefore, has become a new challenge in this field. To tackle this new challenge, there are two issues that need to be addressed. First, Ref. [19] illustrated that the F-measure of their system declined from 0.97 to 0.86 , after increasing the size of test dataset from nearly 2,000 to 20,000 which is defined as large dataset. Therefore, how to maintain a high accuracy of malicious application detection with large-scale data is the first unsolved issue. In addition, when the size of the data increases, it takes more time to process and analyze the data. Therefore, how to decrease the computation time of a large-data analysis is the second issue. Addressing these two issues is non-trivial because there needs to be an accurate solution that can capture malicious mobile applications at a high F-measure and low false positive rate, i.e., it needs to be a solution sufficiently scalable to quickly handle the ever increasing number of mobile applications.

To address these problems, in this study we propose and develop a novel semantic-based approach to represent the feature vectors for detecting malicious mobile applications in large-scale datasets effectively and efficiently. Our method leverages a static analysis to quickly extract features from API calls and permission requests from mobile applications. In addition, we also include the metadata collected from a mobile store as feature. Note that the experimental objects in our study are all related to Android smartphones because the Statistics Portal [6] reports that Android currently has the largest market share for smartphones, accounting for $88 \%$ of all phones in the second quarter of 2018. However, our method can also be used for other smartphone operating systems such as iOS. To develop a scalable and accurate solution, we adopted the state-of-the-art natural language processing (NLP) technique called Doc2Vec [17], which can represent the features in each sentence or document at a semantic level and reduce the feature vectors to any specified dimension. There are many similarities between malicious application detection and NLP because the features obtained from mobile applications and stores can be treated as a form of text processing. Therefore, approaches proposed in the NLP field, such as Doc2Vec, can potentially be used in automating the detection of malicious mobile applications. We built a detection model containing five different types of machine learning algorithms to test the performance of our feature representation method. The experimental result shows that our proposed method contribute to improving the accuracy of all five of the machine learning algorithms in a balanced manner.

In summary, our major contributions include the following:

- We propose a novel approach that can conducts the feature representation for quickly identifying malicious mobile applications from a large volume of data with high accuracy. Note that this approach will likely enhance the performance of many supervised machine learning algorithms.
- We implement our proposed approach in a malicious application detection system by combining a feature extraction component, which obtains static features from applications and metadata, as well as a detection component, which includes five different types of machine learning algorithms.

- We conduct experiments to evaluate the performance of our method. The results show that the best performance of our method can achieve an F-measure of 0.995 with a low false positive rate of $0.48 \%$. Moreover, our method has the ability to process and represent large-scale data in a short time.

The remainder of this paper is organized as follows. Section 2 summarizes the related work and compare them with ours. We describe the high-level overview and details of our proposed method in Section 3. A performance evaluation of our method is given in Section 4. Section 5 discusses the limitation and future work of our system . Finally, conclusions are presented in Section 6.

\section{RELATED WORKS}

There are multiple previous studies focusing on automating the detection of mobile malicious applications using machine or deep learning techniques. In this section, we summarize these previous studies in two categories: machine learning-based approaches and deep learning-based approaches.

\subsection{Machine learning based approaches}

Arp et al. [10] proposed a lightweight method called DREBIN to achieve on-device detection of malicious application. DREBIN uses broad static analysis features, such as requested permissions and API calls, as well as a linear SVM as a detection model. Saracino et al. [24] also developed a host-based malicious application detection system called MADAM. MADAM combines both static and dynamic analysis features and selects the KNN algorithm as a classifier. Sahs et al. [23] used permissions and control flow graphs as features and applied a one-class SVM, which can only be trained by benign applications, which identified malicious applications as anomalies. Jerome et al. [14] extracted opcode sequences from applications and built a detection model with linear SVM. Takahashi et al. [26] designed a scheme to further improve the accuracy of malicious application detection based on the variety types of features consisting of API calls, permissions, category information, and descriptions. Peng et al. [21] proposed an approach to score and rank the risk of applications using probabilistic generative models. Aafer et al. [9] provided insights into malware behavior at the API level and evaluated multiple classifiers to select the best detection model (the KNN classifier) for their system.

These previous studies focused on adjusting the combination of different features and the machine learning algorithm. However, our method can be applied to all types of features and represent the correlations of all these features. Moreover, our represented features can improve the accuracy of all traditional machine learning models without bias.

\subsection{Deep learning based approaches}

Yuan et al. [27] used more than 200 features gained from both static and dynamic analysis results and compared deep belief networks with traditional machine learning models such as SVM and C4.5. 


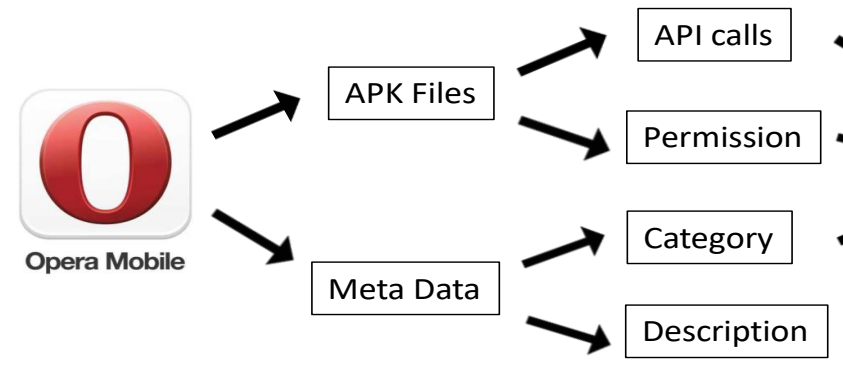

Sec 3.2 Data Collection and Pre-processing

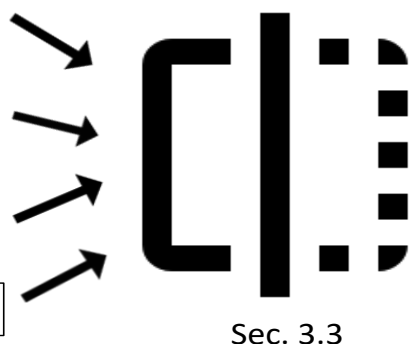

Feature Representation

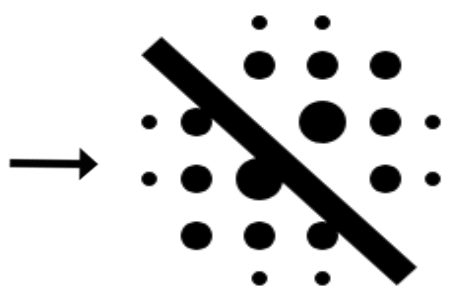

Sec. 3.4

Detection Model

Figure 1: An overview of our methodology.

McLaughlin et al. [19] developed a system to detect Android malware using deep convolutional neural networks. Their features are based on raw opcode sequences. Su et al. [25] proposed an approach called DroidDeep to identify Android malicious applications using Deep Belief Network. They apply the static analysis to extract features from applications quickly so that their method can adapt to a large scale of real-world dataset. Li et al. [18] used a Weight-Adjusted Deep Learning to address the detection of Android malware. Their approach can achieve the same accuracy as previous works using only 237 features. Hou et al. [13] designed a deep learning framework called Deep4MalDroid to enhance Android malware detection. The system adopted by Deep4MalDroid refers to graphs as features and stacked autoencoders as detection models.

The deep learning techniques adopted by the above studies implement representative learning and transfer raw features to weighted features; however, our feature representation method not only represents features but also reduces the dimension of the output feature vectors quickly without lowering the accuracy.

\section{METHODOLOGY}

In this section, we provide an overview of our methodology and detail its four steps: data collection, pre-processing, feature representation, and the detection model.

\subsection{Overview}

Figure 1 presents an overview of our methodology, which consists of four steps. First, in data collection, a crawler is used to collect data from the Opera Mobile Store [4]. Second, the data pre-processing step uses a static analysis to extract useful information from the Android application package (APK) files. Third, the feature representation step encodes the information from the pre-processed data into a semantic-aware feature vector. Fourth, the detection model step preforms an evaluation confirming whether our feature representation method improves the accuracy of malicious application detection and selects the most suitable detection model to fit the detection problem.

\subsection{Data Collection and Pre-processing}

We built a crawler to download APK files from the Opera Mobile Store. In addition, we created a Hypertext Markup Language
(HTML) parser to simultaneously collect metadata from the distribution pages of these APK files. Since the metadata can simply be stored in a database, it is convenient to obtain the categories and descriptions by querying the database. The category can be directly used as a feature; however, the description is raw text and needs to be transformed into an informative feature. We adopted the approach described in Ref. [12] to complete this task. This approach contains three stages as follows.

(1) The data pre-processing stage. Usable words need to be selected for latent Dirichlet allocation (LDA) [11] from the descriptions. First, we checked both the language and the format of the descriptions to remove non-English descriptions and non-textual items, i.e., numbers, HTML tags, web links, and email addresses. Then, we discarded all stop words, such as "is," "am," and "the," and consolidated variant forms of a word into a common form (i.e., word stemming); for example, converting "running" into "run." Finally, we counted the number of words in the description and descriptions containing less than ten words were removed.

(2) The topic model generation stage. We processed the words in the remaining descriptions using LDA. We extracted a total of 300 topics from these descriptions, with a topic proportion threshold of 0.05 and a maximum of four topics per entry. Note that we chose 300 for the number of topics because, according to the MALLET documentation [2], a performance of 200400 can be reasonably fine grained. As a result, this process outputs several (maximum four) topic numberproportion value pairs.

(3) The cluster generation stage. Using k-means [15], the APKs were classified into clusters, according to the topic numberproportion value pairs for each description. We set the number of categories to 12 , which is identical to the number of categories used in the Opera Mobile Store.

The APK file is a compressed file that includes source codes and setting files such as classes.dex and AndroidManifest.xml. It is necessary to conduct an analysis of the APK files to extract information for further analysis. There are two major types of APK analyses for this purpose: static and dynamic. In this paper, we use a static analysis to accomplish the information extraction because such an analysis is lightweight and suitable for our large-scale dataset. We decompressed the APK files and then used Dedexer [1] to convert classes.dex to a Java source code from which the API calls could 


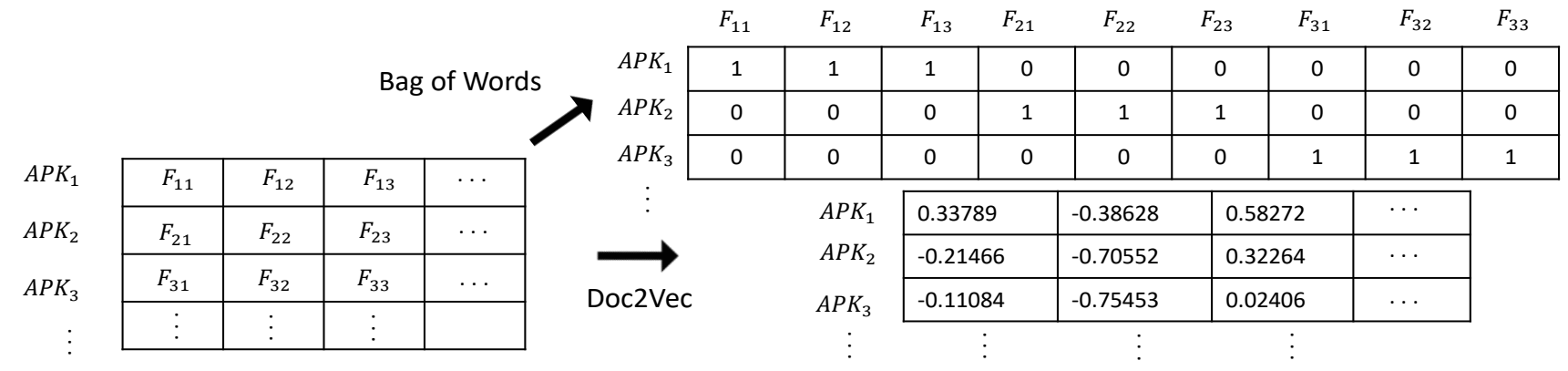

Figure 2: The examples of feature vectors encoded by bag of words and Doc2Vec.

be extracted. After decompressing the APK files, the permission requests can easily be acquired from AndroidManifest.xml.

\subsection{Feature Representation}

Doc2Vec is an extended version of the Word2Vec algorithm [20]. Before introducing Doc2Vec, we explain the principle of Word2Vec in advance.

Word2Vec. Skip-gram models are one of the approaches of Word2Vec that are able to predict the words surrounding a given word. We denote a given word and its nearby words as $w_{I}$ and $w_{O}$, respectively. The goal of a skip-gram model is to determine the maximum value of the following log probability:

$$
\arg \max \log P\left(w_{\mathrm{O} 1}, w_{\mathrm{O} 2}, w_{\mathrm{O} 3} \ldots, w_{\mathrm{OC}} \mid w_{I}\right)
$$

where $C$ is the size of the training context. A larger value of $C$ indicates the addition of more training samples, which can result in a higher accuracy, but leads to a longer training time. Using the softmax function, the above probability can be defined as

$$
P\left(w_{O 1}, w_{O 2}, \ldots, w_{O C} \mid w_{I}\right)=\prod_{c=1}^{C} \frac{\exp \left(v_{w_{O c}}^{\prime T} \cdot v_{w_{I}}\right)}{\sum_{w_{v} \in V} \exp \left(v_{w_{v}}^{\prime T} \cdot v_{w_{I}}\right)}
$$

where $V$ is the number of words in the vocabulary. As $V$ is usually enormous $\left(10^{5}-10^{7}\right.$ terms), Equation 2 suffers from the cost of computing all the surrounding words in the vocabulary. To accelerate this computation, Negative Sampling are used to replace Equation 2 as follows:

$$
E=\log \sigma\left(v_{w_{O}}^{\prime T} \cdot v_{w_{I}}\right)+\sum_{v \in V_{N S}} \sigma\left(-v_{v}^{\prime T} \cdot v_{w_{I}}\right)
$$

Where $\sigma(x)=1 /(1+\exp (-x)), V_{N S}$ indicates that the nearby words, $w_{O}$ to the noise distribution $P_{n}(w)$ are selected using logistic regression. The noise distribution defined in Word2Vec can be described as follows:

$$
P_{n}(w)=\frac{U(w)^{\frac{3}{4}}}{\sum_{v=1}^{V} U\left(w_{v}\right)^{\frac{3}{4}}}
$$

Where $U(w)$ is the frequency of the words. By adopting Negative Sampling, Word2Vec needs to calculate the probability of only $5 \sim$ 20 samples obtained from the noise distribution, rather than all the samples. Word2Vec is implemented by using a single hidden layer neural network. To update the neural network based on the above formulation, the maximum value of Equation 1 can be determined efficiently.

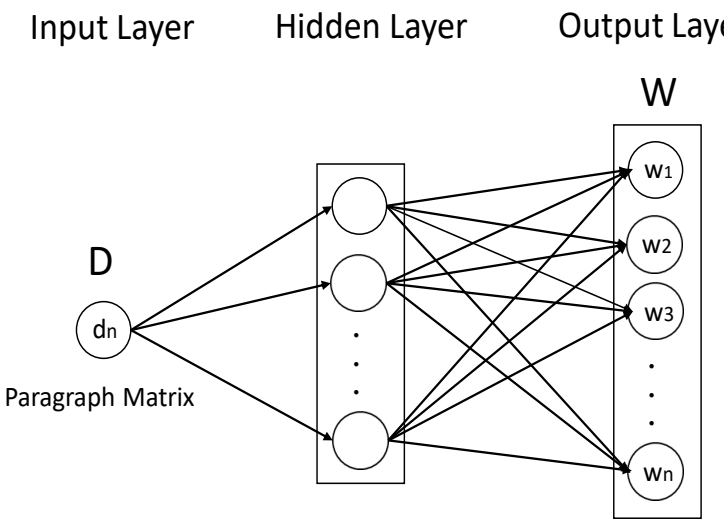

Figure 3: The architecture of Doc2Vec (DBOW).

Doc2Vec. There are two different types of approaches in Doc2Vec: the distributed memory model (DM) and the distributed bag-ofwords (DBOW) model. The DM is designed to predict the next word in the sentence using the input of the word sequences and the paragraph IDs, whereas DBOW ignores the order of words and predicts words that are randomly sampled from the target input paragraph. Note that we only use DBOW models for our feature representation method, because the information described in Section 3.2 does not have order relations. Figure 3 presents the architecture of Doc2Vec (DBOW). DBOW is very similar to the skipgram model in Word2Vec. We can define DBOW by only replacing the $w_{I}$ to $d_{I}$ :

$$
\arg \max \log P\left(w_{\mathrm{O} 1}, w_{\mathrm{O} 2}, w_{\mathrm{O} 3} \ldots, w_{\mathrm{OA}} \mid d_{I}\right)
$$

where $d_{I}$ is a given paragraph ID and $A$ is the number of words in the given paragraph. The rest of the updating approach in neural network is the same as the skip-gram model. We applied the software framework presented in Ref. [22] to implement DBOW in our feature representation method. In this study, we treat the APK ID as a paragraph ID and consider the information extracted from each APK file to be the words in a given paragraph. We trained Doc2Vec with a learning rate of 0.025 for 20 epochs.

Figure 2 shows examples of feature vectors encoded by DBOW and Doc2Vec, wherein $F$ indicates the APK feature. The DBOW 
Table 1: Dataset by category

\begin{tabular}{lrrr}
\hline Category & Benign & Malicious & Total \\
\hline Business \& finance & 3779 & 268 & 4047 \\
Communication & 2114 & 323 & 2437 \\
E-books & 2784 & 479 & 3263 \\
Entertainment & 14138 & 2453 & 16591 \\
Games & 12090 & 2603 & 14693 \\
Health & 1536 & 228 & 1764 \\
Languages \& translators & 734 & 41 & 775 \\
Multimedia & 2422 & 567 & 2989 \\
Organizers & 1300 & 87 & 1387 \\
Ringtone & 327 & 132 & 459 \\
Theme skins & 5276 & 5059 & 10335 \\
Travel \& maps & 2545 & 445 & 2990 \\
\hline Total & 49045 & 12685 & 61730 \\
\hline
\end{tabular}

model encodes the features with 1 or 0 , indicating whether or not the APK file possesses this feature. This type of method generates a high dimension of sparse feature vectors. Moreover, all the encoded features are independent and, thus, are not correlated with each other. Conversely, our method is capable of producing a low dimension of dense feature vectors containing semantic-based information. The feature vectors outputted by our method are the weight of the neural network. Therefore, it is easy to specify the dimension of the represented feature vector by setting the number of hidden layers. Our method can represent the APKs in the form of the inherent relations in each feature, while compressing the feature vector to any size.

\subsection{Detection Model}

We used the Python library scikit-learn [5] to implement the machine learning algorithms in our detection model since this library is efficient and widely used. Considering the diversity of machine learning methods, we selected five different types of standard supervised learning methods, i.e., SVM, KNN, multi-layer perceptron (MLP), logistic regression (LR), and AdaBoost (AB). Some of these algorithms have been adopted by the previous studies described in Section 2. To determine the best machine learning algorithm and parameters, we used our labeled dataset to test all the selected models using classifiers and parameters. The detailed model selection process and its results are presented in Section 4.

\section{EVALUATION}

\subsection{Dataset}

During the period from January to September 2014, we collected 87,182 APK files. We excluded files from which permission requests could not be successfully extracted, because permission requests are included in the informative input for our mobile application detection schemes. We then checked the APK files using VirusTotal [8] to determine whether they were benign or malicious applications. VirusTotal is an online verification service that can evaluate the risk of an APK file using the analysis results of multiple engines from different security vendors. If the APK file was marked in one
Table 2: Dataset by cluster

\begin{tabular}{lrrr}
\hline Cluster & Benign & Malicious & Total \\
\hline 1 & 3574 & 934 & 4508 \\
2 & 3883 & 889 & 4772 \\
3 & 3945 & 976 & 4921 \\
4 & 5247 & 1206 & 6453 \\
5 & 4317 & 1174 & 5491 \\
6 & 3820 & 1077 & 4897 \\
7 & 3474 & 919 & 4393 \\
8 & 5337 & 2091 & 7428 \\
9 & 4104 & 811 & 4915 \\
10 & 4346 & 832 & 5178 \\
11 & 3496 & 818 & 4314 \\
12 & 3502 & 958 & 4460 \\
\hline Total & 49045 & 12685 & 61730 \\
\hline
\end{tabular}

or more of the analysis results provided by the security vendors, we considered the APK file to be a malicious application. Note that adware was not flagged as malicious because most adware are designed to display advertisements. Moreover, because labeled data are necessary for our analysis and test, we removed the APK files that VirusTotal failed to handle in advance. Following the above process, we obtained a dataset of 61,730 APK files, consisting of 49,045 benign and 12,685 malicious files. The statistics of the APK files for each category are shown in Table 1 . The statistics of the cluster classified by the method described in Section 3.2 are presented in Table 2. We share our dataset to all the research communities for the purpose of both improvement research and reproducible study. The details of data download is described on our website ${ }^{2}$.

\subsection{Evaluation Method}

We treated $70 \%$ of the labeled data as the training dataset, while the remaining $30 \%$ were used as the test dataset. Furthermore, we used a grid search to optimize each machine learning model. To optimize the machine learning algorithms, we specified a set of carefully chosen values for each parameter in the machine learning algorithms. For example, for MLP, we set the parameter " $n \_$neighbors" to a set of values: $3,4,5,6$, and 7 . Then, we evaluated the machine learning algorithms with different parameters using a 10-fold cross-validation.

To measure the accuracy of the various supervised learning algorithms, we employed five widely used metrics: accuracy, precision, recall, false positive rate (FPR), and F-measure. The definitions of these metrics are related to the following intermediate measures.

- True Positive (TP): the number of records correctly predicted as positive by a classifier.

- False Positive (FP): the number of records incorrectly predicted as positive by a classifier.

- True negative (TN): the number of records correctly predicted as negative by a classifier.

- False negative (FN): the number of records incorrectly predicted as negative by a classifier.

\footnotetext{
${ }^{2}$ http://mobilesec.nict.go.jp
} 
Table 3: Performance of our feature representation method.

\begin{tabular}{|c|c|c|c|c|c|c|c|c|}
\hline Feature Dimension & Machine Learning & F-measure & Accuracy $(\%)$ & Precision(\%) & Recall(\%) & $\mathrm{FPR}(\%)$ & Train(s) & Test(s) \\
\hline \multirow{5}{*}{500} & $\mathrm{AB}$ & $0.976 \pm 0.002$ & $99.03 \pm 0.07$ & $97.64 \pm 0.19$ & $97.65 \pm 0.31$ & $2.35 \pm 0.31$ & 599.95 & 0.88 \\
\hline & MLP & $0.993 \pm 0.002$ & $99.7 \pm 0.06$ & $99.2 \pm 0.52$ & $99.47 \pm 0.26$ & $0.52 \pm 0.26$ & 24.75 & 0.11 \\
\hline & $\mathrm{KNN}$ & $0.968 \pm 0.001$ & $98.7 \pm 0.06$ & $97.03 \pm 0.29$ & $96.67 \pm 0.17$ & $3.3 \pm 0.17$ & 619.23 & 1.6 \\
\hline & LR & $0.994 \pm 0.001$ & $99.74 \pm 0.03$ & $99.36 \pm 0.16$ & $99.37 \pm 0.1$ & $0.63 \pm 0.1$ & 10.22 & 0.04 \\
\hline & SVM & $0.99 \pm 0.001$ & $99.57 \pm 0.02$ & $98.79 \pm 0.15$ & $99.14 \pm 0.09$ & $0.86 \pm 0.09$ & 86.69 & 24.2 \\
\hline \multirow{5}{*}{100} & $\mathrm{AB}$ & $0.972 \pm 0.002$ & $98.85 \pm 0.09$ & $97.25 \pm 0.43$ & $97.19 \pm 0.47$ & $2.81 \pm 0.47$ & 113.04 & 0.25 \\
\hline & MLP & $0.994 \pm 0.002$ & $99.73 \pm 0.07$ & $99.18 \pm 0.55$ & $99.54 \pm 0.42$ & $0.46 \pm 0.42$ & 15.91 & 0.08 \\
\hline & $\mathrm{KNN}$ & $0.975 \pm 0.002$ & $98.95 \pm 0.07$ & $97.57 \pm 0.27$ & $97.37 \pm 0.2$ & $2.63 \pm 0.02$ & 0.26 & 98.04 \\
\hline & LR & $0.992 \pm 0.001$ & $99.66 \pm 0.04$ & $99.11 \pm 0.15$ & $99.27 \pm 0.06$ & $0.73 \pm 0.06$ & 1.65 & 0.008 \\
\hline & SVM & $0.993 \pm 0.001$ & $99.72 \pm 0.024$ & $99.18 \pm 0.12$ & $99.48 \pm 0.07$ & $0.52 \pm 0.07$ & 10.14 & 3.25 \\
\hline \multirow{5}{*}{50} & $\mathrm{AB}$ & $0.98 \pm 0.002$ & $99.17 \pm 0.07$ & $97.78 \pm 0.28$ & $98.19 \pm 0.19$ & $1.81 \pm 0.19$ & 56.9 & 0.18 \\
\hline & MLP & $0.995 \pm 0.001$ & $99.79 \pm 0.06$ & $99.47 \pm 0.38$ & $99.52 \pm 0.4$ & $0.48 \pm 0.4$ & 18.47 & 0.07 \\
\hline & $\mathrm{KNN}$ & $0.978 \pm 0.19$ & $99.08 \pm 0.08$ & $97.55 \pm 0.3$ & $98.00 \pm 0.21$ & $2.0 \pm 0.21$ & 0.14 & 24.06 \\
\hline & LR & $0.992 \pm 0.001$ & $99.67 \pm 0.05$ & $99.11 \pm 0.15$ & $99.27 \pm 0.14$ & $0.73 \pm 0.14$ & 0.63 & 0.009 \\
\hline & SVM & $0.994 \pm 0.001$ & $99.77 \pm 0.04$ & $99.30 \pm 0.14$ & $99.56 \pm 0.1$ & $0.44 \pm 0.1$ & 4.9 & 1.54 \\
\hline
\end{tabular}

Therefore, the accuracy is the percentage of the test label data that are correctly identified by a classifier. i.e.

$$
\text { Accuracy }=\frac{T P+T N}{n}
$$

The precision is the probability that predicted positive records are correctly classified. i.e.

$$
\text { Precision }=\frac{T P}{T P+F P}
$$

The recall is the probability that the record related to each class is correctly classified. i.e.

$$
\text { Recall }=\frac{T P}{T P+F N}
$$

The FPR is the probability that a negative record is incorrectly considered to be a positive record by a classifier.

$$
F P R=\frac{F P}{F P+T N}
$$

The F-measure is the harmonic mean of the precision and the recall and can evaluate the balance of the two.

$$
F-\text { measure }=2 \cdot \frac{\text { Recall } \cdot \text { Precision }}{\text { Recall }+ \text { Precision }}
$$

\subsection{Evaluation Result}

Table 3 shows the performance evaluation results for our feature representation method. All the results are presented in the form of the average and the standard deviation. The feature dimension encoded by DBOW is 34,995 in our dataset. Our proposed method can reduce the feature dimension by determining the size of the hidden layer of the neural network. In this evaluation, we selected three types of low-level dimensions, 50,100, and 500, to represent the high dimension created by DBOW.

For each type of feature dimension, we evaluated the five types of machine learning algorithms applied in our detection model. Our proposed method can achieve a high F-measure range from 0.968 to 0.995 , with a low FPR of $0.48 \% \sim 3.3 \%$. Note that our feature representation method can improve the accuracy of many machine learning algorithms without any bias. The best performance of the

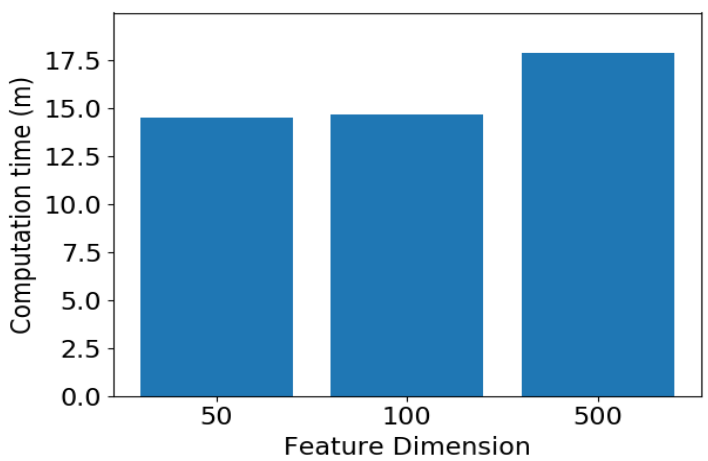

Figure 4: Computation Time.

five machine learning algorithms we tested was achieved by the MLP classifier, because MLP is also designed according to the neural network architecture and is able to update its weights based on the features represented by our method. We used grid search to build our MLP with four hidden layers. The numbers of neural networks in each hidden layer are 100,100,100, and 10, respectively.

The standard deviation of all the evaluation results in each machine learning algorithm is exceptionally low, indicating that a machine learning algorithm based on our method can reliably produce stable results. By reducing the feature dimension from 500 to 50 , the training and test times of all the tested machine learning algorithms dropped significantly, i.e.,., in the case of the $\mathrm{AB}$ algorithm, the training time changed from $599.95 \mathrm{~s}$ to $56.9 \mathrm{~s}$. However, we observed that the evaluation result for 50 dimensions was better than that for other dimensions. Therefore, decreasing the feature dimensions will not lead to a degradation of the performance.

Figure 4 presents the running time of our method. The computation time of each feature dimension is range from $14.5 \mathrm{~min}$ to $17.9 \mathrm{~min}$. In other words, our method is capable of representing and reducing the feature dimension of our large-scale dataset in 
Table 4: Comparison with other previous works.

\begin{tabular}{|c|c|c|c|c|c|c|}
\hline Category & Feature Dimension & F-Measure & Accuracy $(\%)$ & Precision $(\%)$ & Recall(\%) & $\mathrm{FPR}(\%)$ \\
\hline Our proposed Method (MLP) & 50 & 0.995 & 99.79 & 99.47 & 99.52 & 0.48 \\
\hline Pervious work based on machine learning [26] & 2,288 & 0.855 & 94.15 & 87.12 & 84.02 & 3.22 \\
\hline Comparison test using deep learning [16] & 128 & 0.839 & 93.27 & 83.87 & 83.86 & 16.13 \\
\hline
\end{tabular}

less than $18 \mathrm{~min}$. This low computation time enables the detection model to be quickly updated, so as to efficiently discover malicious applications from mobile app stores.

Note that we do not present the effectiveness of the features in this paper. Because our proposed method is different from the traditional feature selection which can compute the importance score of each feature and select the best features based on that. The feature vectors represented by our method are not related to a individual feature and are inextricably connected with other features at the semantic level. Therefore, It is extremely difficult to distinguish which features are more effective in the represented feature vectors. If there is any little effective or ineffective features, our method can weaken or eliminate the influence of these features during the representing process.

Table 4 shows a comparison result with other previous studies. We compared our best detection result (MLP) with those of the other two studies. One is conducted by Ref. [26] and applies feature selection and machine learning technology to identify malicious applications in an Android app store. They adopted SVM-REF for feature selection to reduce the feature dimension and ran SVMREF 11 times for the test, because the performance and size of the reduced feature dimension are different each time. On average, their method reduced the feature dimension from 34,995 to 2,288 , while reaching an F-measure of 0.855 and a FPR of $3.22 \%$.

We also performed a comparison test using convolutional neural network (CNN) [16], a deep learning technology that has been used in many previous studies in both security and NLP and produces good results. The CNN in our test is implemented by TensorFlow [7], which is an open-source machine learning library. We adjusted and set the CNN parameters to a learning rate of 0.001 and a mini-batch of 16 , and the model was then trained for 20 epochs. This CNN has a feature representing ability similar to our method and can embed any size of feature vector in 128 dimensions. Its F-measure and FPR are 0.839 and $16.13 \%$, respectively. Note that the previous study and the comparison test mentioned above were evaluated using exactly the same dataset and features as our study. Compared to these two studies, our proposed method shows excellent performance in terms of both feature dimension reduction and accuracy.

In summary, using a large-scale dataset for a performance evaluation, we illustrated that our method can not only enhance the capability of malicious application detection by achieving a high Fmeasure of 0.995 with a low FPR of $0.48 \%$, but can also drive down the computation time of the feature representation to less than $18 \mathrm{~min}$. Therefore, a detection method based on our feature representation method is suitable for effectively and efficiently detecting malicious applications in large-scale datasets.

\section{DISCUSSION}

This section discusses some limitations of our proposed method and possible solutions to be implemented in future studies.

Data pre-processing. In the data pre-processing step, we applied a static analysis to extract API calls and permission requests from mobile applications. This static analysis cannot cope with native code or dynamic loading in some applications. This is because such programs are executed at the running time and it is difficult to extract such programs from static source codes. A dynamic analysis is capable of capturing such features; however, dynamic analyses suffer from increased analysis processing times. Such an analysis needs to analyze each application for nearly $120 \mathrm{sec}$. It also takes time to restore the operating system after analyzing an application. Therefore, a dynamic analysis is not suitable for a scalable analysis. In our study, we developed a scalable method based on a static analysis without sacrificing accuracy.

Evasion. Machine learning-based approaches are vulnerable to data poisoning attacks. An adversary can deceive the training process by poisoning the benign training samples in the dataset. For example, harmless codes mimicking malicious behaviors can be injected to ensure that some sensitive APIs will rarely be found in the benign samples. If poisoned data are used in the training of the machine learning algorithm, the classifier will not be able to identify malicious applications by leveraging these sensitive APIs as distinctive features. However, our proposed method can build feature vectors in a semantic-based manner, which means that the features are not individual and are mutually related to each other. Therefore, it is more difficult for an adversary to confuse training samples at the semantic level.

Dependence on APK analysis. As mentioned in Section 3.2, part of the input to our proposed method is from the analysis result of the APK file and how the number of sensitive APIs related to malicious behaviors captured by a static or dynamic analysis impacts the performance of the detection method. There are many factors that can lead to this limitation. For example, the malicious applications can use a timer or a type of event to trigger malicious behaviors after the maximum execution time set by the dynamic analysis, while other types of malicious behaviors can be injected into a native code that is executed at the running time. Therefore, it is very difficult to obtain all malicious behaviors using only one analysis method. Note that we do not apply a dynamic analysis in the data pre-processing step due to the analysis processing time. However, our method is designed to represent and reduce the feature dimension to improve the performance of machine learning-based detections. We believe that our evaluation result provides valuable feedback for both static and dynamic analyses and that a security analyst can use our result to adjust their own static or dynamic settings.

Application to other mobile application stores. While this study only used data collected from the Opera Mobile Store, we intend 
to extend our investigation to other app stores in the future. Note that applications and metadata in other app stores are very similar to those in the Opera Mobile Store. Because the static features used by our method depend only on the applications and metadata, it is easy to apply our method to other app stores, such as the iOS App Store or Android third-party app stores.

\section{CONCLUSION}

In this paper, we proposed and developed a novel and semanticaware approach that can enhance the accuracy of identifying malicious mobile applications in a large-scale dataset and effectively reduce the analysis processing running time. We built a crawler and HTML parser to collect a large dataset from a mobile application store and then applied a static analysis to extract features from the application behavior. We also selected five different types of machine learning algorithms for an evaluation test. The experimental results illustrate a best detection performance with an F-measure of 0.995 and a low FPR of $0.48 \%$. In addition, the computation time of our analysis can be decreased to a short time. Moreover, the result of a comparison test shows that our method has an excellent performance compared to other studies in terms of its effectiveness and efficiency.

\section{REFERENCES}

[1] [n. d.]. Dedxer. http://dedexer.sourceforge.net/.

[2] [n. d.]. MALLET Documentation. https://www.cs.cmu.edu/afs/cs.cmu.edu/ project/cmt-40/Nice/Urdu-MT/code/Tools/POS/postagger/mallet_0.4/doc/ documentation.html.

[3] [n. d.]. McAfee Labs Threats Report June 2018. https://www.mcafee.com/ enterprise/en-us/assets/reports/rp-quarterly-threats-jun-2018.pdf.

[4] [n. d.]. Opera Mobile Store - Bemobi. http://android.oms.apps.bemobi.com/.

[5] [n. d.]. scikit-learn:machine learning in Python. http://scikit-learn.org/stable/.

[6] [n. d.]. The Statistics Portal. https://www.statista.com/statistics/266136/ global-market-share-held-by-smartphone-operating-systems/.

[7] [n. d.]. TensorFlow. https://www.tensorflow.org.

[8] [n. d.]. VirusTotal- Free Online Virus, Malware and URL Scanner. https://www. virustotal.com

[9] Yousra Aafer, Wenliang Du, and Heng Yin. 2013. DroidAPIMiner: Mining APILevel Features for Robust Malware Detection in Android. In Security and Privacy in Communication Networks - 9th International ICST Conference, SecureComm 2013, Sydney, NSW, Australia, September 25-28, 2013, Revised Selected Papers. 86-103.

[10] Daniel Arp, Michael Spreitzenbarth, Malte Hubner, Hugo Gascon, and Konrad Rieck. 2014. DREBIN: Effective and Explainable Detection of Android Malware in Your Pocket. In 21st Annual Network and Distributed System Security Symposium, NDSS 2014, San Diego, California, USA, February 23-26, 2014.

[11] David M. Blei, Andrew Y. Ng, and Michael I. Jordan. 2003. Latent Dirichlet Allocation. Journal of Machine Learning Research 3 (2003), 993-1022.

[12] Alessandra Gorla, Ilaria Tavecchia, Florian Gross, and Andreas Zeller. 2014 Checking app behavior against app descriptions. In 36th International Conference on Software Engineering, ICSE '14, Hyderabad, India - May 31 - June 07, 2014. 1025-1035.

[13] Shifu Hou, Aaron Saas, Lifei Chen, and Yanfang Ye. 2016. Deep4MalDroid: A Deep Learning Framework for Android Malware Detection Based on Linux Kernel System Call Graphs. In 2016 IEEE/WIC/ACM International Conference on Web Intelligence - Workshops, WI 2016 Workshops, Omaha, NE, USA, October 13-16, 2016. 104-111.

[14] Quentin Jérome, Kevin Allix, Radu State, and Thomas Engel. 2014. Using opcodesequences to detect malicious Android applications. In IEEE International Conference on Communications, ICC 2014, Sydney, Australia, June 10-14, 2014. 914-919.

[15] J.Macqueen. 1967. Some methods for classification and analysis of multivariate observations. In In 5-th Berkeley Symposium on Mathematical Statistics and Probability. 281-297.

[16] Yoon Kim. 2014. Convolutional Neural Networks for Sentence Classification. In Proceedings of the 2014 Conference on Empirical Methods in Natural Language Processing, EMNLP 2014, October 25-29, 2014, Doha, Qatar, A meeting of SIGDAT, a Special Interest Group of the ACL. 1746-1751.

[17] Quoc V. Le and Tomas Mikolov. 2014. Distributed Representations of Sentences and Documents. In Proceedings of the 31th International Conference on Machine
Learning, ICML 2014, Beijing, China, 21-26 June 2014. 1188-1196.

[18] Wenjia Li, Zi Wang, Juecong Cai, and Sihua Cheng. 2018. An Android Malware Detection Approach Using Weight-Adjusted Deep Learning. In 2018 International Conference on Computing, Networking and Communications, ICNC 2018, Maui, HI, USA, March 5-8, 2018. 437-441.

[19] Niall McLaughlin, Jesús Martínez del Rincón, BooJoong Kang, Suleiman Y. Yerima, Paul C. Miller, Sakir Sezer, Yeganeh Safaei, Erik Trickel, Ziming Zhao, Adam Doupé, and Gail-Joon Ahn. 2017. Deep Android Malware Detection. In Proceedings of the Seventh ACM on Conference on Data and Application Security and Privacy, CODASPY 2017, Scottsdale, AZ, USA, March 22-24, 2017. 301-308.

[20] Tomas Mikolov, Ilya Sutskever, Kai Chen, Gregory S. Corrado, and Jeffrey Dean. 2013. Distributed Representations of Words and Phrases and their Compositionality. In Advances in Neural Information Processing Systems 26: 27th Annual Conference on Neural Information Processing Systems 2013. Proceedings of a meeting held December 5-8, 2013, Lake Tahoe, Nevada, United States. 3111-3119.

[21] Hao Peng, Christopher S. Gates, Bhaskar Pratim Sarma, Ninghui Li, Yuan Qi, Rahul Potharaju, Cristina Nita-Rotaru, and Ian Molloy. 2012. Using probabilistic generative models for ranking risks of Android apps. In the ACM Conference on Computer and Communications Security, CCS'12, Raleigh, NC, USA, October 16-18, 2012. 241-252.

[22] Radim Řehůřrek and Petr Sojka. 2010. Software Framework for Topic Modelling with Large Corpora. In Proceedings of the LREC 2010 Workshop on New Challenges for NLP Frameworks. ELRA, Valletta, Malta, 45-50. http://is.muni.cz/publication/ 884893/en.

[23] Justin Sahs and Latifur Khan. 2012. A Machine Learning Approach to Android Malware Detection. In 2012 European Intelligence and Security Informatics Conference, EISIC 2012, Odense, Denmark, August 22-24, 2012. 141-147.

[24] Andrea Saracino, Daniele Sgandurra, Gianluca Dini, and Fabio Martinelli. 2018. MADAM: Effective and Efficient Behavior-based Android Malware Detection and Prevention. IEEE Trans. Dependable Sec. Comput. 15, 1 (2018), 83-97.

[25] Xin Su, Dafang Zhang, Wenjia Li, and Kai Zhao. 2016. A Deep Learning Approach to Android Malware Feature Learning and Detection. In 2016 IEEE Trustcom/BigDataSE/ISPA, Tianjin, China, August 23-26, 2016. 244-251.

[26] Takeshi Takahashi and Tao Ban. 2019. Android Application Analysis Using Machine Learning Techniques. Springer International Publishing, Cham, 181-205. https: //doi.org/10.1007/978-3-319-98842-9_7

[27] Zhenlong Yuan, Yongqiang Lu, Zhaoguo Wang, and Yibo Xue. 2014. Droid-Sec: deep learning in android malware detection. In ACM SIGCOMM 2014 Conference, SIGCOMM'14, Chicago, IL, USA, August 17-22, 2014. 371-372. 